\title{
Effect of Cherenkov radiation on the jitter of solitons in the driven underdamped Frenkel-Kontorova model
}

\author{
I. I. Soloviev, ${ }^{1,2, *}$ N. V. Klenov, ${ }^{2,3}$ A. L. Pankratov, ${ }^{2,4, \dagger}$ E. Il'ichev, ${ }^{5}$ and L. S. Kuzmin ${ }^{2,6}$ \\ ${ }^{1}$ Skobeltsyn Institute of Nuclear Physics, Moscow State University, Moscow 119991, Russia \\ ${ }^{2}$ Laboratory of Cryogenic Nanoelectronics, Nizhny Novgorod State Technical University, 603950 Nizhny Novgorod, Russia \\ ${ }^{3}$ Physics Department, Moscow State University, Moscow 119991, Russia \\ ${ }^{4}$ Institute for Physics of Microstructures of RAS, 603950 Nizhny Novgorod, Russia \\ ${ }^{5}$ Institute of Photonic Technology, D-07702 Jena, Germany \\ ${ }^{6}$ Chalmers University of Technology, SE-41296 Goteborg, Sweden
}

(Received 6 December 2012; revised manuscript received 9 April 2013; published 5 June 2013)

\begin{abstract}
The effect of complex dynamics of solitons on the output noise of the system (thermal jitter) is studied in the frame of the driven underdamped Frenkel-Kontorova model. In contrast to the continuous case, we have observed a dramatic splash of the jitter. It is demonstrated that this jitter increase is related to the joining of an initial soliton with the one generated by large amplitude oscillations of the Cherenkov radiation tail, which results in the establishment of a unified soliton structure.
\end{abstract}

DOI: 10.1103/PhysRevE.87.060901

PACS number(s): 05.45.Yv, 74.40.-n, 05.40.-a, 85.25.Cp

In recent years the problem of energy dissipated by digital circuits became of importance for further progress in digital technology. The currently demonstrated specific energy dissipation per elementary operation is of the order of $10^{6} \mathrm{kT}$, where $k$ is the Boltzmann constant and $T$ is the temperature. However, the thermodynamic threshold per logic operation, known as the Landauer limit, is equal to $k T \ln 2[1,2]$. To achieve this limit it is necessary to minimize the losses during information processing. A possible approach here is the use of low loss motion of solitary waves for data bit transfer and reversible computation schematics for logic operations.

A broad variety of applications in physics, chemistry, and biology (see $[3,4]$ ) is described by the discrete FrenkelKontorova (FK) model or its continuous analog-the sineGordon (SG) equation. A couple of examples of modern devices used for information processing described by the FK model are as follows. In magnetic domain wall racetrack memory [5], the magnetic domains in nanowires representing data bits can be considered as solitons in the FK model. This device combines the low cost of hard disk drives and the high performance and reliability of solid-state memory. Another example is superconducting electronic devices based on Josephson junctions. Recently it has been experimentally demonstrated that reversible superconductor digital circuits based on underdamped Josephson junctions can operate with extremely low energy dissipation about or even below the Landauer limit [6-9]. Soliton solutions of the FK model correspond to magnetic flux quanta that represent data bits in the circuits. In addition, a number of devices on the basis of superconducting circuits for field and current sensors $[10,11]$ and readout systems for applications in optical communications, quantum cryptography, quantum-optical studies, and radio astronomy [12-16] have been proposed and tested.

Digital technology requires the synchronization of events that correspond to operations with data bits. Since physical

\footnotetext{
*igor.soloviev@gmail.com

†alp@ipmras.ru
}

representation of the bits relates to solitons in the mentioned devices, one of the important parameters limiting their performance is deviation of the soliton propagation time caused by thermal fluctuations. The standard deviation of this time is called jitter. The importance of the effect of jitter in the digital circuits was first understood in Ref. [17] and later studied both analytically and numerically in Refs. [18-20]. Formulas, based on the driven sine-Gordon model with damping [21] predict that the jitter accumulates as the square root of the system length or even faster [10]. This was confirmed experimentally for the discrete damped system [22]. At the same time, it has been shown recently that for the continuous case one can expect a substantial reduction of jitter increase with system length due to Lorentz contraction of the solitons [23].

Because of the growing interest in the use of underdamped FK systems for information processing, the results of Ref. [23] inspired us to study the influence of discreteness of the model (leading, e.g., to Cherenkov radiation) on the jitter accumulation process. In this Rapid Communication we demonstrate that complex nonlinear dynamics of solitons in the FK model [24-29] strongly affects the output noise of the system.

Let us consider the driven underdamped FK model [3] with noise, representing a chain of underdamped Josephson junctions, in the similar range of parameters as in Refs. [10,23]:

$$
\ddot{\phi}_{n}-\left(\phi_{n-1}-2 \phi_{n}+\phi_{n+1}\right) / l+\sin \phi_{n}=i_{n}+i_{n f}-\alpha \dot{\phi}_{n},
$$

where $\phi$ is the Josephson phase, index $n$ denotes the number of the junction, and the dot means the time derivative. This chain is analogous to coupled pendula with coupling constant $1 / l$, damping $\alpha$, driving force $i_{n}$, and fluctuational term $i_{n f}$. Equation (1) describes an array of the junctions connected with normalized inductances $l=2 e I_{c} L / \hbar$, where $I_{c}$ is the critical current of the Josephson junction, $L$ is the inductance, $e$ is the electron charge, $\hbar$ is the Planck constant, $\alpha=\omega_{p} / \omega_{c}$ is the damping parameter, $\omega_{p}=\left(2 e I_{c} / \hbar C\right)^{1 / 2}$ is the plasma frequency, $C$ is the junction capacitance, $\omega_{c}=2 e I_{c} R_{N} / \hbar$ is the characteristic Josephson frequency, and $R_{N}$ is the 
normal state resistance. Time is normalized to $\omega_{p}^{-1}$. Since the quiescent soliton occupies approximately $\sim 4 / \sqrt{l}$ junctions (or $4 \lambda_{J}$ [30], where $\lambda_{J}$ is the Josephson penetration depth), we have considered the range $l \geqslant 0.5$ where discreteness affects its motion noticeably. The junctions are fed by constant current $i_{n}=I / I_{c}$. The thermal fluctuational current $i_{n f}$ is modeled by the white Gaussian noise with zero mean, and the correlation function $\left\langle i_{n f}(t) i_{n f}(t+\tau)\right\rangle=2 \alpha \gamma \delta(\tau)$, with dimensionless noise intensity $\gamma=I_{T} / I_{c}, I_{T}=2 e k T / \hbar$.

The switching time of the system is defined as the time of soliton passage through the considered chain when the last junction reaches a certain value above an unstable equilibrium point of its potential profile. Then, the mean value and the standard deviation (jitter) $\sigma$ of this time are calculated by averaging over 1000-10 000 realizations. As an estimate it is convenient to start with the variance of switching time $\sigma_{J}^{2}$ for a single junction in two limiting cases-high and low damping [30]:

$$
\begin{aligned}
& \sigma_{J}^{2} \approx \bar{\tau} \gamma i^{\prime-2} \quad \text { for } \alpha \gg 1, \\
& \sigma_{J}^{2} \approx \bar{\tau} \gamma i^{\prime-8 / 5} \omega_{p}^{-2 / 5} \quad \text { for } \alpha \ll 1,
\end{aligned}
$$

where $i^{\prime}$ is the time derivative of total current through the junction and $\bar{\tau}$ is the average time during which the junction phase is close to an unstable equilibrium point overcoming a barrier of its potential profile. We have used $\bar{\tau}$ in Eqs. (2) as a fitting parameter which can be obtained from numerical calculations of the junction dynamics. It is worth noting that for both cases the variance is inversely proportional to the rate of the current growth and proportional to $\bar{\tau}$.

In our calculations we fixed the initial shape of a soliton. The results of numerical calculation of Eq. (1) for jitter accumulation with increase of the system length are shown in Fig. 1(a) by dots; analytical approximations of calculated data by Eq. (2) are shown by solid curves. Good agreement indicates that we can consider the junctions as sources of independent fluctuations described by Eq. (2). A decrease of the damping parameter corresponds to an increase in the rate of all processes, including the rate of current growth $i^{\prime}$ and the decrease of $\bar{\tau}$, which in turn corresponds to jitter decrease [see the inset of Fig. 1(a)].

The jitter accumulation for different values of current $i_{n}$ is shown in Fig. 1(b). The deviation of the jitter from the square root law means unequal influence of fluctuations on dynamics of the junctions. According to Eqs. (2) this corresponds to different $i^{\prime}$ and $\bar{\tau}$ that follow from Lorenz contraction [23] (or broadening) during acceleration (or deceleration) of solitons. The steady soliton velocity is defined by the "power balance" of perturbing terms grouped in the right-hand side of Eq. (1), $i_{n} \int_{0}^{t_{p}} \dot{\phi} d t=\alpha \int_{0}^{t_{p}} \dot{\phi}^{2} d t$, where $t_{p}$ is the time during which the soliton passes the $n$th junction. Soliton acceleration and duration of its accelerated motion depends on $i_{n}$ and $\alpha$, and can be estimated from calculations provided in Ref. [10] (see Fig. 5 there) and in Ref. [23]. Contrary to the work [23] this acceleration was not taken into account in the evaluation of jitter in Ref. [10]. Our results confirm the possibility of jitter suppression [23] for the discrete case [see Fig. 1(b)].

Total jitter of the system versus current $i_{n}$ is calculated for various values of damping $\alpha$ [see Fig. 2]. The number of junctions in the system is $N=30$. Jitter suppression corresponds

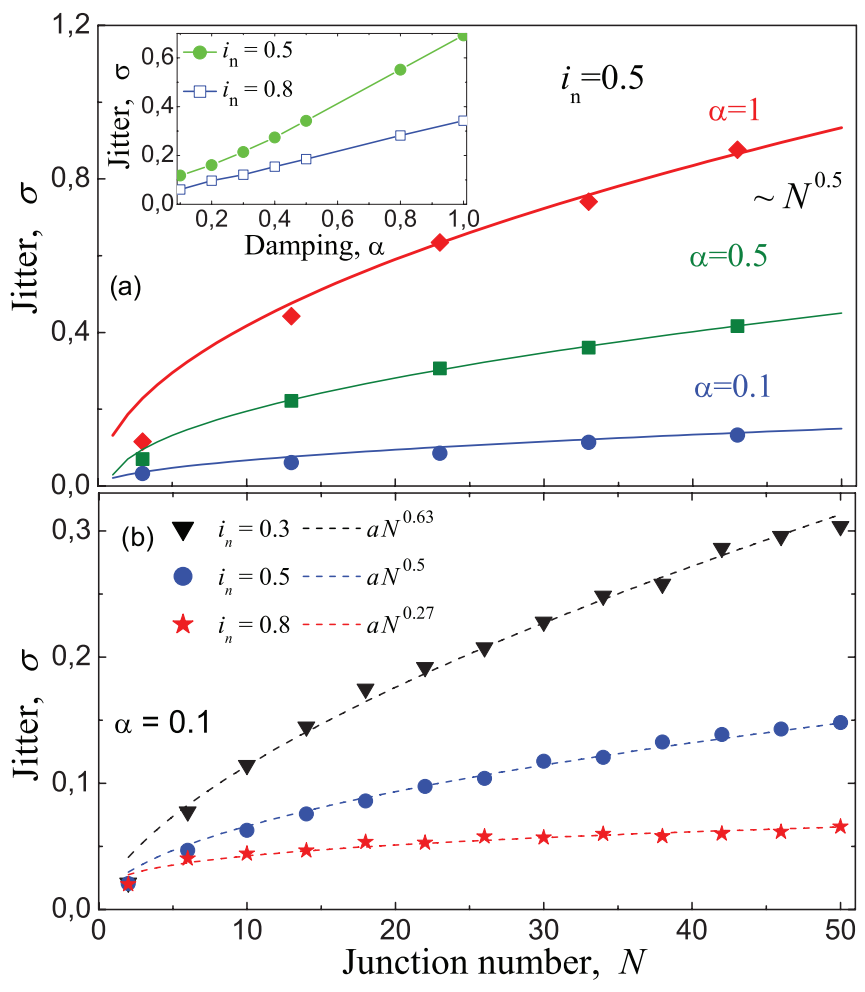

FIG. 1. (Color online) Jitter accumulation versus number of junctions $N$ for (a) different values of damping $\alpha$ [dots, numerical calculations; solid curves, analytical approximations (2)] and (b) different currents (dots, numerical calculations; dashed curves, fitting by function $y=a N^{x} ; a$ is a fitting coefficient). The inset of (a) shows total jitter decrease with damping for the systems consisting of $N=30$ junctions. Coupling inductance $l=2.5$; noise intensity $\gamma=10^{-3}$.

to described relations between the soliton velocity, $i_{n}$, and $\alpha$. Increase of the jitter in the vicinity of the critical current value, $i_{n}=1$, can be explained by the growing influence of thermally activated processes on passing the unstable equilibrium point. Rapid growth of the jitter for the small

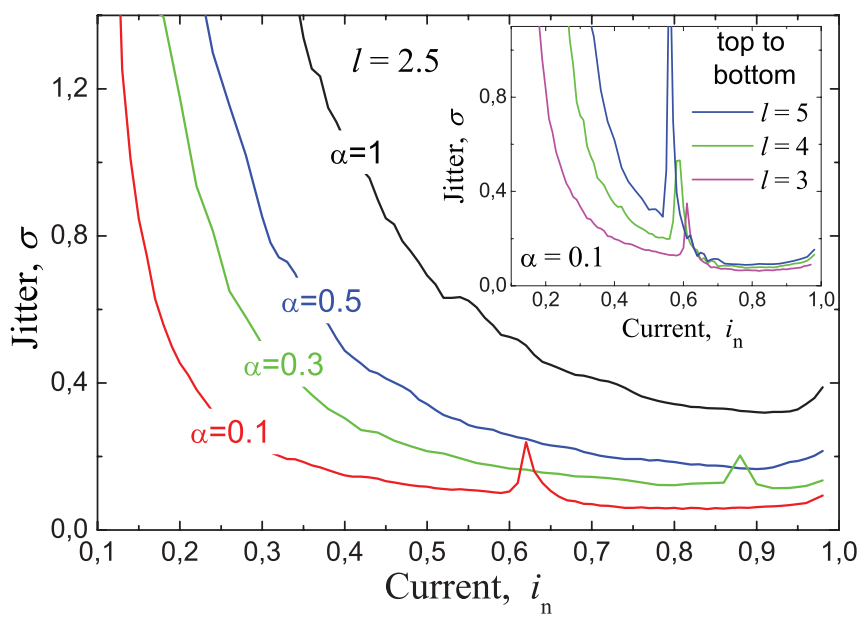

FIG. 2. (Color online) Total jitter versus current $i_{n}$ for the systems consisting of $N=30$ junctions and parameters shown in the figures. The inset shows the jitter versus current curves for the systems with different discreteness. Noise intensity $\gamma=10^{-3}$. 
current values is caused by discreteness of the system (see the inset of Fig. 2). It corresponds to the pinning of the soliton in a well of spatially periodic Peierls-Nabarro potential which reflects noninvariance of the system to arbitrary translation of the soliton along the chain [3,24]. In the ideal situation without noise, the depinning current can be calculated analytically following the work [31]. Jitter splashes in the middle of these curves for small values of the damping parameter $(\alpha=0.1,0.3)$ are rather unexpected. They cannot be predicted or explained in the frame of the conventional approach for the jitter evaluation based on the SG equation. Their explanation in the frame of the FK model is as follows.

The soliton behaves as a quasiparticle with its own characteristic mass and velocity, so it is possible to consider the problem of excitation of linear waves by a fast moving soliton as Cherenkov radiation. It is well known that this radiation cannot exist in the SG model. However, in the discrete model the soliton can be accompanied by a steady wave in its reference frame, which can be treated as a tail of Cherenkov radiation [3,24]. In the case of small damping this might lead to instability of a fast soliton described in Ref. [28]. An example of the dynamics of a phase kink, which corresponds to the soliton in the FK model, is shown in Figs. 3(a)-3(c). If the current value is larger than the threshold
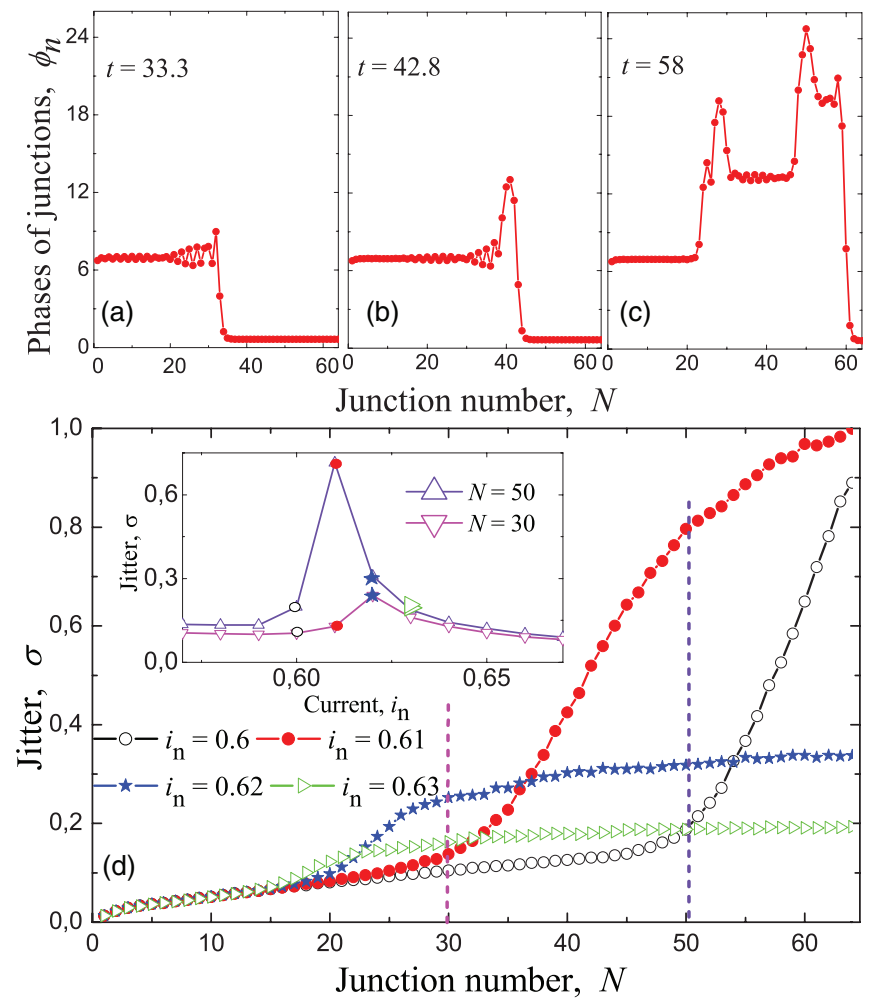

FIG. 3. (Color online) Dynamics of phase kink, corresponding to the soliton in the FK model, during system transition from locked to running mode at different moments of time (a), (b) and (c). The system consists of $N=64$ junctions fed by current $i_{n}=0.61, l=$ 2.5, $\alpha=0.1$. (d) Jitter accumulation in this system for the same and neighbor current values. Noise intensity $\gamma=10^{-3}$. The inset of (d) presents jitter versus current for two different lengths of the system $(N=30$ and 50). Dashed vertical lines with corresponding colors cross the jitter curves in (d) at these junction numbers. one, a discrete breather can be captured in the soliton tail and then decays into a soliton-antisoliton pair (the corresponding $2 \pi$ kink-antikink on the phase plots). The initial soliton and the new one join in a single soliton structure with double topological charge. This transformation of oscillation modes is repeated then for both solitons and antisolitons, forcing the formation of an expanding domain, within which the phases of junctions are running with nearly constant velocity close to the maximum one $i_{n} / \alpha$. Boundaries of the domain are the multi- $2 \pi$ phase kink structures propagating with maximum available velocity for solitons $c=\omega_{p} \lambda_{J}$. The current threshold value of the system transition from locked to running mode and the corresponding threshold velocity of a soliton can be obtained from the work [28].

Jitter accumulation corresponding to the presented dynamics is shown in Fig. 3(d) by the red curve with solid circles. It is seen that the jitter grows rapidly during the transition process in the narrow range of the currents [see the inset of Fig. 3(d)]. Different jitter splashes for the different lengths of the system shown in the inset are explained by different finite lengths and
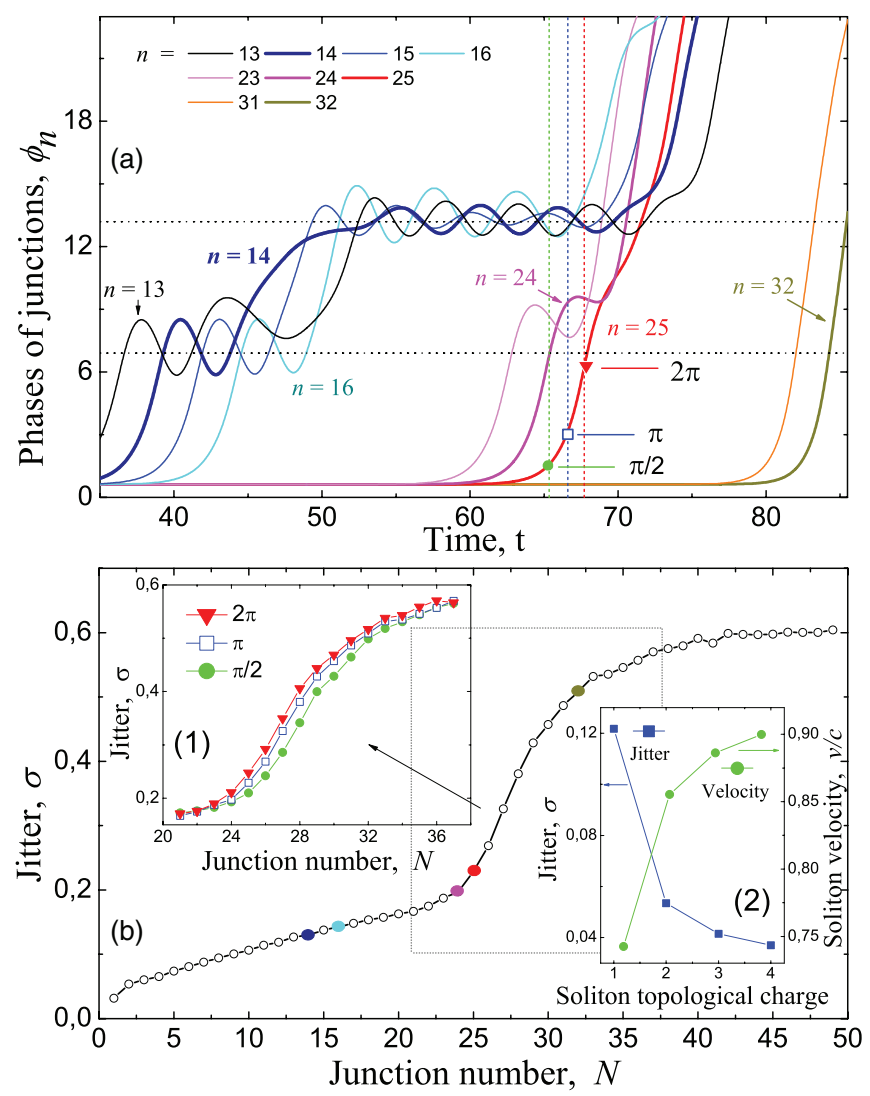

FIG. 4. (Color online) (a) Dynamics of junction phases during the transition process in the system consisting of $N=48$ junctions with high discreteness $(l=4), i_{n}=0.58$, and $\alpha=0.1$. The second and the third equilibrium phase points are shown by horizontal dotted lines. (b) Jitter accumulation with the system length. Inset (1) shows jitter accumulation curves calculated at different phase levels $(\pi / 2, \pi$, and $2 \pi$ ) in the range of junctions highlighted by the frame. Inset (2) presents jitter and soliton velocity versus soliton topological charge for the system consisting of $N=32$ junctions coupled by inductances $l=2.5 . i_{n}=0.5, \alpha=0.1$. Noise intensity $\gamma=10^{-3}$. 
starting points of the transition process which depend on the current value for certain $l$ and $\alpha$.

The dynamics of junction phases during the transition process is presented in Fig. 4(a). To make the transition process more pronounced we have considered a system with higher discreteness $l=4$. Stable equilibrium points of junction phase are defined by the applied current $\phi_{\mathrm{st}}=\arcsin \left(i_{n}\right)+2 \pi m$, where $m$ is an integer. Soliton propagation corresponds to $2 \pi$ phase jump $(m=0 \rightarrow 1)$. The transition process starts from junction number $n=14$ when its phase jumps from the second stable point $(m=1)$ to the third one $(m=2)$ (both are shown in the figure by horizontal dotted lines). Propagation of a newly created soliton and antisoliton corresponds to the similar additional jumps of phases of the subsequent and previous junctions. Still our calculation of jitter accumulation shows that the appearance of this soliton-antisoliton pair does not lead to the jitter enhancement [see Fig. 4(b)].

We calculated the jitter accumulation stopping the calculation when the phase of currently final junction in the system reached the various determined levels. The values of these levels $(\pi / 2, \pi$, and $2 \pi)$ are marked on the phase curve of junction number $n=25$ in Fig. 4(a). The corresponding jitter curves are shown in inset (1) of Fig. 4(b). There is almost no discrepancy of these curves up to junction number $n=24$. This means that fluctuations do not affect the dynamics of the currently final junction in the system while it passes these phase levels because they are far from its unstable equilibrium point. The discrepancy of these curves for systems consisting of a larger number of junctions indicates the impact of fluctuations from the nearest previous junctions in the jitter which follows from the fact that these junctions linger near maximum of their potential barrier (above $m=1$ ) at these moments. The jitter curves converge with the end of formation of the multi- $2 \pi$ phase kink boundary structure of the expanding running phase domain. According to Eq. (1) jitter accumulation is minimum for this high speed structure and the considered additional impact in output phase noise is vanishing [see the phase curve for junction number $n=32$, Fig. 4(a), and note the slope near the end of the jitter accumulation curve in Fig. 4(b)].

In the current range below the threshold one the jitter can be minimized further by using a preliminarily formed soliton structure with multiple topological charge. Such structure is dynamically stable in the discrete system and propagates with a higher velocity than a single charge soliton [3]. With an increase of the topological charge the minimum current value, below which the structure is unstable, increases also. This leads to a tradeoff between jitter minimization and margins of available currents. For the middle current value $i_{n}=0.5$, jitter and soliton velocity versus soliton topological charge are presented in inset (2) of Fig. 4(b).

In conclusion we have shown that contrary to the continuous system counterpart [23] the high values of driving force (or current in Josephson systems) in the discrete underdamped FK model can lead to a significant increase of soliton jitter, which is related to joining of an initial soliton with the one generated by large amplitude oscillations of the Cherenkov radiation tail, which results in the establishment of a unified soliton structure. The margins of available driving force in the model corresponding to operation with individual solitons is defined by the height of Peierls-Nabarro relief and threshold value of the force switching the system from locked to running mode. If the running mode is undesirable due to avalanche of the system, the jitter can be additionally suppressed by using soliton structures with multiple topological charge which are dynamically stable in the discrete case.

This work is supported by Russian Ministry of Science (Grants No. 11.G34.31.0029, No. 14.513.11.0009, and No. 8746), Dynasty Foundation, and Human Capital Foundation.
[1] R. Landauer, IBM J. Res. Dev. 3, 183 (1961).

[2] C. Bennett, IBM J. Res. Dev. 17, 525 (1973).

[3] O. M. Braun and Yu. S. Kivshar, The Frenkel-Kontorova Model: Concepts, Methods, and Applications (Springer-Verlag, Berlin, 2004).

[4] Y. S. Kivshar and B. A. Malomed, Rev. Mod. Phys. 61, 763 (1989).

[5] S. S. P. Parkin, M. Hayashi, and L. Thomas, Science 320, 190 (2008).

[6] V. K. Semenov, G. V. Danilov, and D. V. Averin, IEEE Trans. Appl. Supercond. 13, 938 (2003).

[7] V. K. Semenov, G. V. Danilov, and D. V. Averin, IEEE Trans. Appl. Supercond. 17, 455 (2007).

[8] J. Ren, V. K. Semenov, Y. A. Polyakov, D. V. Averin, and J.-S. Tsai, IEEE Trans. Appl. Supercond. 19, 961 (2009).

[9] J. Ren and V. K. Semenov, IEEE Trans. Appl. Supercond. 21, 780 (2011).

[10] A. Fedorov, A. Shnirman, G. Schon, and A. KidiyarovaShevchenko, Phys. Rev. B 75, 224504 (2007).
[11] A. Herr, A. Fedorov, A. Shnirman, E. Il'ichev, and G. Schon, Supercond. Sci. Technol. 20, S450 (2007).

[12] V. K. Semenov and D. V. Averin, IEEE Trans. Appl. Supercond. 13, 960 (2003).

[13] D. V. Averin, K. Rabenstein, and V. K. Semenov, Phys. Rev. B 73, 094504 (2006).

[14] T. Ortlepp, M. Hofherr, L. Fritzsch, S. Engert, K. Ilin, D. Rall, H. Toepfer, H.-G. Meyer, and M. Siegel, Opt. Express 19, 18593 (2011).

[15] H. Terai, T. Yamashita, S. Miki, K. Makise, and Z. Wang, Opt. Express 20, 20115 (2012).

[16] M. A. Tarasov, L. S. Kuzmin, V. S. Edelman, N. S. Kaurova, M. Yu. Fominskii, and A. B. Ermakov, JETP Lett. 92, 416 (2009).

[17] A. V. Rylyakov and K. K. Likharev, IEEE Trans. Appl. Supercond. 9, 3539 (1999).

[18] A. L. Pankratov and B. Spagnolo, Phys. Rev. Lett. 93, 177001 (2004).

[19] V. K. Semenov and A. Inamdar, IEEE Trans. Appl. Supercond. 15, 435 (2005). 
[20] A. V. Gordeeva and A. L. Pankratov, Appl. Phys. Lett. 88, 022505 (2006).

[21] D. W. McLaughlin and A. C. Scott, Phys. Rev. A 18, 1652 (1978).

[22] H. Terai, Z. Wang, Y. Hishimoto, S. Yorozu, A. Fujimaki, and N. Yoshikawa, Appl. Phys. Lett. 84, 2133 (2004).

[23] A. L. Pankratov, A. V. Gordeeva, and L. S. Kuzmin, Phys. Rev. Lett. 109, 087003 (2012).

[24] A. V. Ustinov, M. Cirillo, and B. A. Malomed, Phys. Rev. B 47, 8357 (1993).

[25] A. V. Ustinov, M. Cirillo, B. H. Larsen, V. A. Oboznov, P. Carelli, and G. Rotoli, Phys. Rev. B 51, 3081 (1995).
[26] O. M. Braun, A. R. Bishop, and J. Röder, Phys. Rev. Lett. 79, 3692 (1997).

[27] A. V. Ustinov, B. A. Malomed, and S. Sakai, Phys. Rev. B 57, 11691 (1998)

[28] O. M. Braun, B. Hu, and A. Zeltser, Phys. Rev. E 62, 4235 (2000).

[29] K. G. Fedorov, M. V. Fistul, and A. V. Ustinov, Phys. Rev. B 84, 014526 (2011).

[30] K. K. Likharev, Dynamics of Josephson Junctions and Circuits (Gordon and Breach, New York, 1986).

[31] G. Derks, A. Doelman, S. A. van Gils, and H. Susanto, SIAM J. Appl. Dyn. Syst. 6, 99 (2007). 